\title{
Field of a Horizontal Magnetic Dipole in the Presence of a Magnetoplasma Halfspace
}

\author{
G. Tyras, ${ }^{1}$ A. Ishimaru, ${ }^{2}$ and H. M. Swarm ${ }^{2}$
}

(Received December 26, 1962; revised April 4, 1963)

\begin{abstract}
In the present paper we consider the problem of a horizontal magnetic dipole situated in or out of a lossy magnetoplasma halfspace when the magnetostatic field is parallel to the axis of the dipole. The rigorous formulation of the problem is carried to the point where the determination of the pertinent boundary coefficients remains to be a straightforward but not a simple algebraic process. Due to the prohibitive algebraic complexity involved in the explicit finding of the boundary coefficients, a high frequency approximation is introduced and the approximate form of the boundary coefficients found. The field integrals are evaluated in the air region for the condition when the observation point is at a large distance from the source. The results are applied to finding the radiation pattern in the air due to a magnetic dipole situated near the lower edge of the ionosphere. It is found that the most important correction due to the earth's magnetic field occurs near the interface and parallel to the axis of the dipole.
\end{abstract}

\section{Introduction}

The problem of a horizontal electric and magnetic dipole in air and in the presence of a conducting, homogeneous, and isotropic halfspace was originally formulated and solved by Sommerfeld [1926]. Extending the work of Sommerfeld to a conducting halfspace, Moore [1951] and also Baños and Wesley [1953] solved the problem of the horizontal electric dipole while Wait and Campbell [1953] and Wait [1959] solved the problem of a horizontal magnetic dipole.

The problem of radiation in free space from sources in magnetoplasma halfspace was first considered by Barsukov [1959]. He obtained expressions for the Poynting vector in air for the cases when the magnetostatic field was normal to the boundary and an electric dipole was either normal or parallel to the boundary. He also presented a numerical example for a vertical dipole at the boundary and for the particular set of conditions considered he found that the plasma's anisotropy manifested itself most strongly in the directivity of the radiation pattern. A similar problem was also considered by Arbel [1960].

In the present paper we shall treat the case of a horizontal magnetic dipole situated either in or out of the magnetoplasma when the magnetostatic field is parallel to the dipole axis. The choice of a horizontal magnetic dipole as the source of the electromagnetic waves was motivated by the fact that such an antenna in the presence of an interface effectively produces both vertically and horizontally polarized waves; thus, both wave polarizations could be studied in a single problem. Moreover, since the electric field lines form circular loops about the axis of a magnetic dipole and the magnetostatic field is parallel to the same, then the alternating electric field is normal to the steady magnetic field and, thus, maximum interaction of the electromagnetic wave with the magnetostatic field could be expected.

\section{Dipole in Magnetoplasma}

\subsection{Rigorous Formulation}

The geometry of the problem is shown in figure 1 . The horizontal plane $z=0$ coincides with the interface between the anisotropic homogeneous plasma and air. For conven-

\footnotetext{
1 Present address: Department of Electrical Engineering, University of Arizona, Tucson, Ariz. On leave from The Boeing Co., Seattle Wash. This work, was carried out when this author was at Boeing.

¿Department of Electrical Engineering, University of Washington, Seattle, Wash.
} 


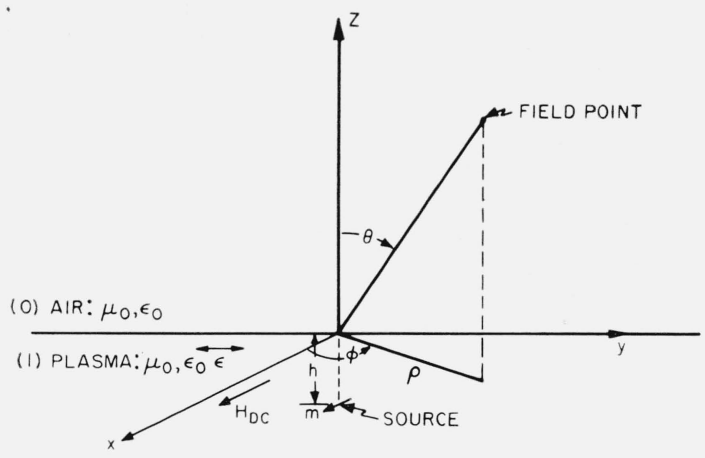

FiguRE 1. Geometry of the problem of a magnetic dipole in magnetoplasma.

ience we shall call the plasma medium (1) and the air medium (0). The plasma, in addition to its anisotropy, may be lossy while the conductivity of the air is zero. Moreover, we assume that both media have the same magnetic inductive capacity of free space, $\mu_{0}$. The factor causing the anisotropy of the plasma, the magnetostatic field $H_{D C}$, is oriented in the positive $x$-direction as well as the source of the electromagnetic waves, the magnetic dipole.

The definition of the present boundary value problem implies the solution to Maxwell's equations subject to the usual boundary conditions at the interface and proper behavior at infinity. In the air region it is convenient to introduce the Hertzian vector potential of the magnetic type whereas in the plasma region it is found to be more convenient to work with the actual field components.

For the purpose of this problem it will be assumed that the source of the electromagnetic waves consists of a small wire loop carrying an electric current $I e^{-i \omega t}$. When the loop is small enough, its electromagnetic effects can be adequately represented by its equivalent dipole moment

$$
\vec{m}=I \cdot \vec{S}
$$

where $\vec{S}$ is the surface vector of the area enclosed by the loop. We localize the source by writing for the magnetic current density

$$
\vec{J}_{m}=\vec{m} \delta(x) \delta(y) \delta(z+h) .
$$

In the magnetoplasma region the magnetic field will satisfy the inhomogeneous vector wave equation

$$
\left(-\vec{\nabla} \times \overrightarrow{\epsilon^{-1}} \vec{\nabla} \times+k_{0}^{2}\right) \vec{H}_{1}=-i \omega \epsilon_{0} m \delta(x) \delta(y) \delta(z+h) \overrightarrow{1}_{x}
$$

where $\overleftrightarrow{\epsilon}$ is the permittivity tensor given by [Tyras and Held, 1959]

and

$$
\stackrel{\epsilon}{\epsilon}=\left(\begin{array}{lll}
\zeta & 0 & 0 \\
0 & \epsilon & i \eta \\
0 & -i \eta & \epsilon
\end{array}\right)
$$

$$
\begin{aligned}
\zeta & =1-\frac{\omega_{p}^{2}}{\omega^{2}+\nu^{2}}+\frac{i \nu \omega_{p}^{2}}{\omega\left(\omega^{2}+\nu^{2}\right)} \\
\epsilon & =1-\frac{\omega_{p}^{2}\left(\omega^{2}-\omega_{H}^{2}+\nu^{2}\right)}{\Delta}+\frac{i \nu \omega_{p}^{2}\left(\omega^{2}+\omega_{H}^{2}+\nu^{2}\right)}{\omega \Delta} \\
\eta & =\frac{\omega_{H} \omega_{p}^{2}\left(\omega^{2}-\omega_{H}^{2}-\nu^{2}\right)}{\omega \Delta}-\frac{i 2 \nu \omega_{H} \omega_{p}^{2}}{\Delta} \\
\Delta & =\left[\left(\omega+\omega_{H}\right)^{2}+\nu^{2}\right]\left[\left(\omega-\omega_{H}\right)^{2}+\nu^{2}\right] \\
\omega_{p}^{2} & =\frac{e^{2} N}{m_{e} \epsilon_{0}} ; \quad \omega_{H}=\frac{-|e| \mu_{0} H_{D C}}{m_{e}}
\end{aligned}
$$


where $N$ is the number of free electrons per cubic meter, $|e|=1.6 \times 10^{-19}$ coulomb is the electronic charge, and $m_{e}=9.1 \times 10^{-31} \mathrm{~kg}$ is the electronic mass.

In the air region we use the Hertzian vector of the magnetic type defined by

$$
\vec{E}=i \omega \mu_{0} \vec{\nabla} \times \vec{\Pi}^{(M)}
$$

and satisfying the homogeneous vector wave equation

$$
\left(\nabla^{2}+k_{0}^{2}\right) \vec{\Pi}^{(M)}=0 .
$$

The formulation of the present boundary value problem can be simplified a great deal by expressing the field components in the magnetoplasma and the air regions in terms of their triple Fourier integral representation in Cartesian coordinates in the transform space as well as in the configuration space. To this end we introduce a triple Fourier transform pair defined by

$$
\begin{aligned}
\overline{\bar{F}}\left(\alpha_{1}, \alpha_{2}, \alpha_{3}\right) & =\frac{1}{(2 \pi)^{3 / 2}} \iint_{-\infty}^{\infty} \int_{-} F(x, y, z) e^{-i\left(\alpha_{1} x+\alpha_{2} y+\alpha_{3} z\right)} d x d y d z \\
F(x, y, z) & =\frac{1}{(2 \pi)^{3 / 2}} \iint_{-\infty}^{\infty} \int_{\bar{F}} \overline{\bar{F}}\left(\alpha_{1}, \alpha_{2}, \alpha_{3}\right) e^{i\left(\alpha_{1} x+\alpha_{2} y+\alpha_{3} z\right)} d \alpha_{1} d \alpha_{2} d \alpha_{3} .
\end{aligned}
$$

In what follows we shall also need the transforms of the derivatives. These can be obtained by integrating by parts where the vanishing of the integrated part is assured providing that the fields have a proper behavior at infinity.

Thus transforming (3) one obtains for the plasma region

$$
\left(\begin{array}{ccc}
\chi k_{0}^{2}-\alpha_{2}^{2}-\alpha_{3}^{2} & \alpha_{1}\left(\alpha_{2}+i \kappa \alpha_{3}\right) & \alpha_{1}\left(\alpha_{3}-i \kappa \alpha_{2}\right) \\
\alpha_{1}\left(\alpha_{2}-i \kappa \alpha_{3}\right) & \chi k_{0}^{2}-\alpha_{1}^{2}-\frac{\chi}{\zeta} \alpha_{3}^{2} & \frac{\chi}{\zeta} \alpha_{2} \alpha_{3}+i \kappa \alpha_{1}^{2} \\
\alpha_{1}\left(\alpha_{3}+i \kappa \alpha_{2}\right) & \frac{\chi}{\zeta} \alpha_{2} \alpha_{3}-i \kappa \alpha_{1}^{2} & \chi k_{0}^{2}-\alpha_{1}^{2}-\frac{\chi}{\zeta} \alpha_{2}^{2}
\end{array}\right)\left(\begin{array}{c}
\overline{\bar{H}}_{x 1} \\
\overline{\bar{H}}_{y 1} \\
\overline{\overline{\bar{H}}}_{z 1}
\end{array}\right)=\frac{-i \omega \epsilon_{0} \chi m}{(2 \pi)^{3 / 2}}\left(\begin{array}{c}
e^{i \alpha_{3} h} \\
0 \\
0
\end{array}\right)
$$

where $^{3}$

$$
\chi=\frac{\epsilon^{2}-\eta^{2}}{\epsilon} ; \quad \kappa=\frac{\eta}{\epsilon}
$$

The system of algebraic equations in (9) can be solved using Cramer's rule. The determinant of the coefficients of the square matrix on the left is found to be

$$
\Delta=\frac{\chi^{2} k_{0}^{2}}{\zeta}\left(\alpha_{3}^{2}-s_{1}^{2}\right)\left(\alpha_{3}^{2}-s_{2}^{2}\right)
$$

where

$$
s_{1,2}^{2}=\frac{1}{2}\left\{(\chi+\zeta) k_{0}^{2}-\frac{\chi+\zeta\left(1-\kappa^{2}\right)}{\chi} \alpha_{1}^{2}-2 \alpha_{2}^{2} \pm \sqrt{\left[(\chi-\zeta) k_{0}^{2}-\frac{\chi-\zeta\left(1-\kappa^{2}\right)}{\chi} \alpha_{1}^{2}\right]^{2}+4 \zeta \kappa^{2} k_{0}^{2} \alpha_{1}^{2}}\right\} .
$$

The result in (12) is not surprising. It is typical of what could be expected from analysis of a double refracting medium like magnetoplasma. We have two modes of propagation characterized by the eigenvalues $s_{1}$ and $s_{2}$ with which terms "ordinary" and "extraordinary" are often associated. These eigenvalues are given by the zeroes of $\Delta$ and are the poles' of the

3 The correspondence between our notation for plasma parameters and that of the magneto-ionic theory [Ratcliff, 1959] is as follows: $\epsilon=1+C U$, $\eta=C Y_{2}, \zeta=1-X / U$. We feel that our notation is more convenient for our purpose. 
field components. We note, for instance, that if $\alpha_{2}=\alpha_{3}=0$ in (11), which is equivalent to saying that the wave does not vary along the $y$ and $z$ coordinates, we obtain for the eigenvalues

$$
\left(\alpha_{1}\right)_{1,2}=k_{0} \sqrt{\epsilon \pm \eta}
$$

which can be recognized as the propagation constants of the right- and left-hand circularly polarized plane waves propagating in the direction of the magnetostatic field. Alternately, if $\alpha_{1}=\alpha_{3}=0$ we find

$$
\begin{aligned}
& \left(\alpha_{2}\right)_{1}=k_{0} \sqrt{\chi} \\
& \left(\alpha_{2}\right)_{2}=k_{0} \sqrt{\zeta}
\end{aligned}
$$

which again can be recognized as plane wave propagation constants transverse to the steady magnetic field. Setting $\alpha_{1}=\alpha_{2}=0$ and solving for $\alpha_{3}$ gives the same results as in (14).

We now proceed with formally solving the system of algebraic equations in (9). Using Cramer's rule and the results of (11) and (12) one obtains

$$
\begin{aligned}
& \overline{\bar{H}}_{x 1}^{(p)}=-\frac{i \omega \epsilon_{0} m}{(2 \pi)^{3 / 2} \chi k_{0}^{2}} \cdot \frac{\Phi_{1}\left(\alpha_{1}, \alpha_{2}, \alpha_{3}\right)}{\left(\alpha_{3}^{2}-s_{1}^{2}\right)\left(\alpha_{3}^{2}-s_{2}^{2}\right)} e^{i \alpha_{3} h} \\
& \overline{\bar{H}}_{y 1}^{(p)}=\frac{i \omega \epsilon_{0} m}{(2 \pi)^{3 / 2} \chi k_{0}^{2}} \cdot \frac{\alpha_{1}\left[\alpha_{2} \Phi_{2}\left(\alpha_{1}, \alpha_{2}, \alpha_{3}\right)-i \kappa \chi \zeta k_{0}^{2} \alpha_{3}\right]}{\left(\alpha_{3}^{2}-s_{1}^{2}\right)\left(\alpha_{3}^{2}-s_{2}^{2}\right)} e^{\imath \alpha_{3} h} \\
& \overline{\bar{H}}_{z 1}^{(p)}=\frac{i \omega \epsilon_{0} m}{(2 \pi)^{3 / 2} \chi k_{0}^{2}} \cdot \frac{\alpha_{1}\left[\alpha_{3} \Phi_{2}\left(\alpha_{1}, \alpha_{2}, \alpha_{3}\right)+i \kappa \chi \zeta k_{0}^{2} \alpha_{2}\right]}{\left(\alpha_{3}^{2}-s_{1}^{2}\right)\left(\alpha_{3}^{2}-s_{2}^{2}\right)} e^{i \alpha_{3} h}
\end{aligned}
$$

where

$$
\begin{aligned}
& \Phi_{1}\left(\alpha_{1}, \alpha_{2}, \alpha_{3}\right)=\left(\chi k_{0}^{2}-\alpha_{1}^{2}\right)\left[\zeta\left(\chi k_{0}^{2}-\alpha_{1}^{2}\right)-\chi\left(\alpha_{2}^{2}+\alpha_{3}^{2}\right)\right]-\kappa^{2} \zeta \alpha_{1}^{4} \\
& \Phi_{2}\left(\alpha_{1}, \alpha_{2}, \alpha_{3}\right)=\zeta \chi k_{0}^{2}-\zeta\left(1-\kappa^{2}\right) \alpha_{1}^{2}-\chi\left(\alpha_{2}^{2}+\alpha_{3}^{2}\right) .
\end{aligned}
$$

The inversion with respect to the $\alpha_{3}$-transform variable can be performed immediately by integrating in the complex $\alpha_{3}$-plane along a path that runs along the real axis then along a circular arc in the upper half-plane for $z+h>0$, and the lower half-plane for $z+h<0$. It can be shown that the contribution from integration along the circular arc vanishes providing $\operatorname{Im}\left\{s_{1,2}\right\}>0$ and the resulting integral is equal to the sum of the residues at the poles $s_{1}$ and $s_{2}$. Furthermore, inverting with respect to the $\alpha_{1}$ - and $\alpha_{2}$-transform variable one obtains the integral representation of the primary excitation as follows:

$$
\begin{aligned}
& H_{x 1}^{(p)}=\frac{\omega \epsilon_{0} m}{8 \pi^{2} \chi k_{0}^{2}} \int_{-\infty}^{\infty}\left\{\frac{\Phi_{1}\left(s_{1}\right) e^{i s_{1}|z+h|}}{s_{1}\left(s_{1}^{2}-s_{2}^{2}\right)}-\frac{\Phi_{1}\left(s_{2}\right) e^{i s_{2}|z+h|}}{s_{2}\left(s_{1}^{2}-s_{2}^{2}\right)}\right\} e^{i\left(\alpha_{1} x+\alpha_{2} y\right)} d \alpha_{1} d \alpha_{2} \\
& H_{\substack{y 1 \\
z+h \gtrless 0}}^{(p)} \frac{i \omega \epsilon_{0} m}{s \pi^{2} \chi k_{0}^{2}} \partial_{x} \int_{-\infty}^{\infty}\left\{\frac{\left[\alpha_{2} \Phi_{2}\left(s_{1}\right) \mp i \kappa \chi \zeta k_{0}^{2} s_{1}\right] e^{i s_{1}|z+h|}}{s_{1}\left(s_{1}^{2}-s_{2}^{2}\right)}-\frac{\left[\alpha_{2} \Phi_{2}\left(s_{2}\right) \mp i \kappa \chi \zeta k_{0}^{2} s_{2}\right] e^{i s_{2}|z+h|}}{s_{2}\left(s_{1}^{2}-s_{2}^{2}\right)}\right\} e^{i\left(\alpha_{1} x+\alpha_{2} y\right)} d \alpha_{1} d \alpha_{2} \\
& H_{\substack{z 1 \\
z+h \gtrless 0}}^{(p)} \frac{i \omega \epsilon_{0} m}{8 \pi^{2} \chi k_{0}^{2}} \partial_{x} \int_{-\infty}^{\infty}\left\{\frac{\left[ \pm s_{1} \Phi_{2}\left(s_{1}\right)+i \kappa \chi \zeta k_{0}^{2} \alpha_{2}\right] e^{\imath s_{1}|z+h|}}{s_{1}\left(s_{1}^{2}-s_{2}^{2}\right)}-\frac{\left[ \pm s_{2} \Phi_{2}\left(s_{2}\right)+i \kappa \chi \zeta k_{0}^{2} \alpha_{2}\right] e^{i s_{2}|z+h|}}{s_{2}\left(s_{1}^{2}-s_{2}^{2}\right)}\right\} e^{i\left(\alpha_{1} x+\alpha_{2} y\right)} d \alpha_{1} d \alpha_{2} .
\end{aligned}
$$

To satisfy the boundary conditions of the problem, we shall also need an appropriate complementary solution of the homogeneous system of (9). From the theory of linear differential equations [Ince, 1956] it follows that the magnetic field satisfies

$$
\left(\alpha_{3}^{2}-s_{1}^{2}\right)\left(\alpha_{3}^{2}-s_{2}^{2}\right) \stackrel{\overrightarrow{\widetilde{H}}}{(c)}=0
$$


where $\alpha_{3}$ in this equation corresponds to the differential operator $i \partial_{2}$. The solution to (18) can be written immediately

$$
\left(\begin{array}{c}
\widetilde{\tilde{H}}_{x 1}^{(c)} \\
\widetilde{\tilde{H}}_{y 1}^{(c)} \\
\widetilde{\widetilde{H}}_{z 1}^{(c)}
\end{array}\right)=\left(\begin{array}{ll}
C_{11} & C_{12} \\
C_{21} & C_{22} \\
C_{31} & C_{32}
\end{array}\right) \quad\left(\begin{array}{l}
e^{-i s_{1} z} \\
e^{-i s_{2} z}
\end{array}\right)
$$

where we discarded solutions with positive exponentials for physical reasons. The coefficients $C_{i j}$ are not all independent. For since $\underset{\widetilde{H^{(c)}}}{\overrightarrow{2}}$ must satisfy the given system the coefficients must be related by

and

$$
\left(\begin{array}{lll}
F_{11}\left(-s_{1}\right) & F_{12}\left(-s_{1}\right) & F_{13}\left(-s_{1}\right) \\
F_{21}\left(-s_{1}\right) & F_{22}\left(-s_{1}\right) & F_{23}\left(-s_{1}\right) \\
F_{31}\left(-s_{1}\right) & F_{32}\left(-s_{1}\right) & F_{33}\left(-s_{1}\right)
\end{array}\right) \quad\left(\begin{array}{l}
C_{11} \\
C_{21} \\
C_{31}
\end{array}\right)=0
$$

$$
\left(\begin{array}{lll}
F_{11}\left(-s_{2}\right) & F_{12}\left(-s_{2}\right) & F_{13}\left(-s_{2}\right) \\
F_{21}\left(-s_{2}\right) & F_{22}\left(-s_{2}\right) & F_{23}\left(-s_{2}\right) \\
F_{31}\left(-s_{2}\right) & F_{32}\left(-s_{2}\right) & F_{33}\left(-s_{2}\right)
\end{array}\right) \quad\left(\begin{array}{l}
C_{12} \\
C_{22} \\
C_{32}
\end{array}\right)=0
$$

where the square matrix elements $F_{i j}\left(s_{1,2}\right)$ correspond to the square matrix of the original system (9) with $\alpha_{3}=-s_{1}$, or $\alpha_{3}=-s_{2}$. Now we express all coefficients $C_{i j}$ in terms $C_{11}$ and $C_{12}$ and normalize obtaining

$$
\begin{aligned}
& C_{21}=-\frac{\omega \epsilon_{0} m \alpha_{1}}{4 \pi \chi k_{0}^{2}}\left[\frac{\alpha_{2} \Phi_{2}\left(s_{1}\right)+i \kappa \chi \zeta k_{0}^{2} s_{1}}{\Phi_{1}\left(s_{1}\right)}\right] A_{1} \\
& C_{31}=-\frac{\omega \epsilon_{0} m \alpha_{1}}{4 \pi \chi k_{0}^{2}}\left[\frac{-s_{1} \Phi_{2}\left(s_{1}\right)+i \kappa \chi \xi k_{0}^{2} \alpha_{2}}{\Phi_{1}\left(s_{1}\right)}\right] A_{1}
\end{aligned}
$$

and

$$
\begin{aligned}
& C_{22}=-\frac{\omega \epsilon_{0} m \alpha_{1}}{4 \pi \chi k_{0}^{2}}\left[\frac{\alpha_{2} \Phi_{2}\left(s_{2}\right)+i_{\kappa} \chi \zeta k_{0}^{2} s_{2}}{\Phi_{1}\left(s_{2}\right)_{1}}\right] A_{2} \\
& C_{32}=-\frac{\omega \epsilon_{0} m \alpha_{1}}{4 \pi \chi k_{0}^{2}}\left[\frac{-s_{2} \Phi_{2}\left(s_{2}\right)+i \kappa \chi \zeta k_{0}^{2} \alpha_{2}}{\Phi_{1}\left(s_{2}\right)}\right] A_{2} .
\end{aligned}
$$

Inverting with respect to the $\alpha_{1}$ and $\alpha_{2}$ transform variables we obtain the complementary solution as follows:

$$
\begin{aligned}
& H_{x 1}^{(c)}=\frac{\omega \epsilon_{0} m}{8 \pi^{2} \chi k_{0}^{2}} \int_{-\infty}^{\infty} \int_{-\infty}^{\infty}\left[A_{1} e^{-i s_{1} z}+A_{2} e^{-i s_{2} z}\right] e^{i\left(\alpha_{1} x+\alpha_{2} y\right)} d \alpha_{1} d \alpha_{2} \\
& H_{y 1}^{(c)}=\frac{1}{2 \pi} \int_{-\infty}^{\infty}\left[C_{21} e^{-i s_{1} z}+C_{22} e^{-i s_{2} z}\right] e^{i\left(\alpha_{1} x+\alpha_{2} y\right)} d \alpha_{1} d \alpha_{2} \\
& H_{z 1}^{(c)}=\frac{1}{2 \pi} \iint_{-\infty}^{\infty}\left[C_{31} e^{-i s_{1} z}+C_{32} e^{-i s_{2} z}\right] e^{i\left(\alpha_{1} x+\alpha_{2} y\right)} d \alpha_{1} d \alpha_{2} .
\end{aligned}
$$

The coefficients $A_{1}$ and $A_{2}$, representing waves reflected from the interface, will be determined uniquely from the boundary conditions.

The Hertz vector potential in the air region must satisfy the wave equation (7). The appropriate solution can be written 


$$
\begin{aligned}
& \Pi_{x}^{(M)}=\frac{\omega \epsilon_{0} m}{8 \pi^{2} \chi k_{0}^{4}} \iint_{-\infty}^{\infty} B_{1} e^{i\left(\alpha_{1} x+\alpha_{2} y+s_{0} z\right)} d \alpha_{1} d \alpha_{2} \\
& \Pi_{z}^{(M)}=\frac{-\omega \epsilon_{0} m}{8 \pi^{2} \chi k_{0}^{4}} \int_{-\infty}^{\infty} \int_{-\infty} B_{2} e^{i\left(\alpha_{1} x+\alpha_{2} y+s_{0} z\right)} d \alpha_{1} d \alpha_{2}
\end{aligned}
$$

where $s_{0}=\left(k_{0}^{2}-\alpha_{1}^{2}-\alpha_{2}^{2}\right)^{1 / 2}$ and we shall require that $\operatorname{Im}\left\{s_{0}\right\}>0$. The coefficients $B_{1}$ and $B_{2}$, representing waves transmitted through the interface, will be determined uniquely from the boundary conditions.

The boundary conditions to be satisfied by the Cartesian components of the field vectors require continuity of the tangential components of the electric and magnetic fields at the interface $z=0$. This implies the following

$$
\begin{aligned}
& k_{0}^{2} \Pi_{x}^{(M)}+\partial_{x}\left(\partial_{x} \Pi_{x}^{(M)}+\partial_{z} \Pi_{z}^{(M)}\right)=H_{x 1} \\
& \partial_{y}\left(\partial_{x} \Pi_{x}^{(M)}+\partial_{z} \Pi_{z}^{(M)}\right)=H_{y 1} \\
& \zeta k_{0}^{2} \partial_{y} \Pi_{z}^{(M)}=\partial_{y} H_{z 1}-\partial_{z} H_{y 1} \\
& \chi k_{0}^{2}\left(\partial_{z} \Pi_{x}^{(M)}-\partial_{x} \Pi_{z}^{(M)}\right)=\left(i \kappa \partial_{y}+\partial_{z}\right) H_{x 1}-i \kappa \partial_{x} H_{y 1}-\partial_{x} H_{z 1} .
\end{aligned}
$$

The above boundary conditions give four equations in four unknowns $A_{1}, A_{2}$, $B_{1}$, and $B_{2}$. It can be shown that this system of four equations can be reduced to just two equations in two unknowns as follows:

where

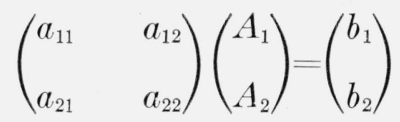

$$
\begin{aligned}
& a_{11,2}=\alpha_{2}\left(s_{1,2}+\chi s_{0}-i \kappa \alpha_{2}\right)+\alpha_{1}^{2}\left(\frac{\chi}{\zeta} s_{1,2}+\chi s_{0}-i \kappa \alpha_{2}\right) \frac{\left[\alpha_{2} \Phi_{2}\left(s_{1,2}\right)+i \kappa \chi \zeta k_{0}^{2} s_{1,2}\right]}{\Phi_{1}\left(s_{1,2}\right)} \\
& +\alpha_{1}^{2} \alpha_{2}(\chi-\zeta) \frac{\left[-s_{1,2} \Phi_{2}\left(s_{1,2}\right)+i \kappa \chi \zeta k_{0}^{2} \alpha_{2}\right]}{\zeta \Phi_{1}\left(s_{1,2}\right)} \\
& \begin{aligned}
& a_{21,2}= \frac{\alpha_{2}\left[\Phi_{1}\left(s_{1,2}\right)-\left(s_{0}^{2}+\alpha_{2}^{2}\right) \Phi_{2}\left(s_{1,2}\right)\right]-i \kappa \chi k_{0}^{2}\left[\zeta s_{1,2}\left(s_{0}^{2}+\alpha_{2}^{2}\right)+s_{0}\left(s_{1,2}^{2}+\alpha_{2}^{2}\right)\right]}{\Phi_{1}\left(s_{1,2}\right)} \\
& b_{1}=\left[\alpha_{2}\left(s_{1}-\chi s_{0}+i \kappa \alpha_{2}\right) \Phi_{1}\left(s_{1}\right)+\alpha_{1}^{2}\left(\frac{\chi}{\zeta} s_{1}-\chi s_{0}+i \kappa \alpha_{2}\right)\left(\alpha_{2} \Phi_{2}\left(s_{1}\right)-i \kappa \chi \zeta k_{0}^{2} s_{1}\right)\right.
\end{aligned} \\
& \left.\quad-\alpha_{1}^{2} \alpha_{2}\left(\frac{\chi}{\zeta}-1\right)\left(s_{1} \Phi_{2}\left(s_{1}\right)+i \kappa \chi \zeta k_{0}^{2} \alpha_{2}\right)\right] \frac{e^{i s_{1} h}}{s_{1}\left(s_{1}^{2}-s_{2}^{2}\right)} \\
& \quad-\left[\alpha_{2}\left(s_{2}-\chi s_{0}+i \kappa \alpha_{2}\right) \Phi_{1}\left(s_{2}\right)+\alpha_{1}^{2}\left(\frac{\chi}{\zeta} s_{2}-\chi s_{0}+i \kappa \alpha_{2}\right)\left(\alpha_{2} \Phi_{2}\left(s_{2}\right)-i \kappa \chi \zeta k_{0}^{2} s_{2}\right)\right. \\
& \left.-\alpha_{1}^{2} \alpha_{2}\left(\frac{\chi}{\zeta}-1\right)\left(s_{2} \Phi_{2}\left(s_{2}\right)+i \kappa \chi \zeta k_{0}^{2} \alpha_{2}\right)\right] \frac{e^{i s_{2} h}}{s_{2}\left(s_{1}^{2}-s_{2}^{2}\right)}
\end{aligned}
$$

$$
\begin{aligned}
b_{2}=-\left\{\alpha_{2}\left[\Phi_{1}\left(s_{1}\right)-\left(s_{0}^{2}+\alpha_{2}^{2}\right) \Phi_{2}\left(s_{1}\right)\right]+i \kappa \chi k_{0}^{2}\left[\zeta s_{1}\left(s_{0}^{2}+\alpha_{2}^{2}\right)-s_{0}\left(s_{1}^{2}+\alpha_{2}^{2}\right)\right]\right\} \frac{e^{i s_{1} h}}{s_{1}\left(s_{1}^{2}-s_{2}^{2}\right)} \\
+\left\{\alpha_{2}\left[\Phi_{1}\left(s_{2}\right)-\left(s_{0}^{2}+\alpha_{2}^{2}\right) \Phi_{2}\left(s_{2}\right)\right]+i \kappa \chi k k_{0}^{2}\left[\zeta s_{2}\left(s_{0}^{2}+\alpha_{2}^{2}\right)-s_{0}\left(s_{2}^{2}+\alpha_{2}^{2}\right)\right]\right\} \frac{e^{i s_{2} h}}{s_{2}\left(s_{1}^{2}-s_{2}^{2}\right)} .
\end{aligned}
$$

Once the coefficients $A_{1}$ and $A_{2}$ are found, the remaining coefficients $B_{1}$ and $B_{2}$ can be determined from (25). In fact we find 


$$
\begin{aligned}
\alpha_{2} B_{1}= & \left\{\alpha_{2}\left[\Phi_{1}\left(s_{1}\right)+\alpha_{1}^{2} \Phi_{2}\left(s_{1}\right)\right]+i \kappa \chi \zeta k_{0}^{2} s_{1} \alpha_{1}^{2}\right\} \frac{A_{1}}{\Phi_{1}\left(s_{1}\right)} \\
& +\left\{\alpha_{2}\left[\Phi_{1}\left(s_{2}\right)+\alpha_{1}^{2} \Phi_{2}\left(s_{2}\right)\right]+i \kappa \chi \zeta k_{0}^{2} s_{2} \alpha_{1}^{2}\right\} \frac{A_{2}}{\Phi_{1}\left(s_{2}\right)}+\left\{\alpha_{2}\left[\Phi_{1}\left(s_{1}\right)+\alpha_{1}^{2} \Phi_{2}\left(s_{1}\right)\right]-i \kappa \chi \zeta k_{0}^{2} s_{1} \alpha_{1}^{2}\right\} \frac{e^{i s_{1} h}}{s_{1}\left(s_{1}^{2}-s_{2}^{2}\right)} \\
& \quad-\left\{\alpha_{2}\left[\Phi_{1}\left(s_{2}\right)+\alpha_{1}^{2} \Phi_{2}\left(s_{2}\right)\right]-i \kappa \chi \zeta k_{0}^{2} s_{2} \alpha_{1}^{2}\right\} \frac{e^{i s_{2} h}}{s_{2}\left(s_{1}^{2}-s_{2}^{2}\right)}
\end{aligned}
$$

$$
\begin{aligned}
\frac{\alpha_{2} s_{0}}{\alpha_{1}} B_{2}=\left\{\alpha _ { 2 } \left[\Phi_{1}\left(s_{1}\right)-\right.\right. & \left.\left.\left(s_{0}^{2}+\alpha_{2}^{2}\right) \Phi_{2}\left(s_{1}\right)\right]-i_{\kappa} \chi \zeta k_{0}^{2} s_{1}\left(s_{0}^{2}+\alpha_{2}^{2}\right)\right\} \frac{A_{1}}{\Phi_{1}\left(s_{1}\right)} \\
& +\left\{\alpha_{2}\left[\Phi_{1}\left(s_{2}\right)-\left(s_{0}^{2}+\alpha_{2}^{2}\right) \Phi_{2}\left(s_{2}\right)\right]-i \kappa \chi \zeta k_{0}^{2} s_{2}\left(s_{0}^{2}+\alpha_{2}^{2}\right)\right\} \frac{A_{2}}{\Phi_{1}\left(s_{2}\right)} \\
& +\left\{\alpha_{2}\left[\Phi_{1}\left(s_{1}\right)-\left(s_{\ominus}^{2}+\alpha_{2}^{2}\right) \Phi_{2}\left(s_{1}\right)\right]+i \kappa \chi \zeta k_{0}^{2} s_{1}\left(s_{0}^{2}+\alpha_{2}^{2}\right)\right\} \frac{e^{i s_{1} h}}{s_{1}\left(s_{1}^{2}-s_{2}^{2}\right)} \\
& -\left\{\alpha_{2}\left[\Phi_{1}\left(s_{2}\right)-\left(s_{0}^{2}+\alpha_{2}^{2}\right) \Phi_{2}\left(s_{2}\right)\right]+i \kappa \chi \zeta k_{0}^{2} s_{2}\left(s_{0}^{2}+\alpha_{2}^{2}\right)\right\} \frac{e^{i s_{2} h}}{s_{2}\left(s_{1}^{2}-s_{2}^{2}\right)} .
\end{aligned}
$$

The explicit determination of the four boundary coefficients $A_{1}, A_{2}, B_{1}$, and $B_{2}$ is now a straightforward although a tedious matter. The results would necessarily be lengthy and probably not very useful. In the next section we shall introduce a high frequency approximation which will simplify the results a great deal without the loss of the essential aspects of the problem.

\subsection{High Frequency Approximation}

Any approximation that we may introduce to simplify the field components is necessarily contingent upon the simplification of the propagation factors in the $z$-direction; i.e., $s_{1}$ and $s_{2}$ given by (12). A useful approximation will prove itself to be one based on the assumption

$$
\left|\frac{e \mu_{0} H_{D C}}{\omega m_{e}}\right|<<1
$$

which can be brought about by either a weak magnetostatic field or by a sufficiently high wave frequency. ${ }^{4}$ Furthermore, for a high enough wave frequency it usually happens that

$$
\frac{\nu}{\omega}<<1
$$

is also satisfied. If these two assumptions hold, it can be shown that the components of the permittivity tensor in (4) can be simplified as follows:

$$
\begin{aligned}
& \chi \sim \epsilon \sim \zeta \\
& \kappa \sim \eta / \zeta \\
& \zeta \sim 1-\left(\frac{\omega_{p}}{\omega}\right)^{2}+i \frac{\nu \omega_{p}^{2}}{\omega^{2}} \\
& \eta \sim \frac{\omega_{H} \omega_{p}^{2}}{\omega^{3}}-i 2 \frac{\nu \omega_{H} \omega_{p}^{2}}{\omega^{4}}
\end{aligned}
$$

where we retained only first order terms in $\omega_{H} / \omega$ and $\nu / \omega$. Consistent with these approximations it can be shown that $s_{1,2}$ in (12) simplifies to

$$
s_{1,2}^{2} \sim s^{2} \pm \kappa k_{1} \alpha_{1}
$$

${ }^{4}$ In the earth's ionosphere, the region of validity of this approximation will be limited to frequencies in excess of $1 \mathrm{Mc} / \mathrm{s}$. 
where $s=\left(k_{1}^{2}-\alpha_{1}^{2}-\alpha_{2}^{2}\right)$ and $k_{1}=\sqrt{\zeta} k_{0}$. Now in regions where $\left|s^{2}\right|>>\left|\kappa k_{1} \alpha_{1}\right|$ we can approximate further by writing

$$
s_{1,2} \sim s \pm \frac{\kappa k_{1} \alpha_{1}}{2 s}
$$

It will appear that last form of $s_{1,2}$ is indeed the only useful one. The usefulness of it is, however, reduced by the fact that it is not valid in the vicinity of $s=0$ which corresponds to the critical angle in the absence of the magnetostatic field.

Introduction of approximation (32) into (27) and (28) results in the following:

where

$$
\begin{aligned}
& a_{11}=c_{1}-\kappa d_{1} \\
& a_{12}=c_{2}+\kappa d_{2} \\
& a_{22}=e_{1}-\kappa f_{1} \\
& a_{21}=e_{2}+\kappa f_{2}
\end{aligned}
$$

Moreover

$$
\begin{gathered}
b_{1}=-\frac{\zeta k_{1}^{2}}{s}\left\{\alpha_{2}\left(s-\zeta s_{0}\right)+\frac{i \kappa}{2}\left[\alpha_{1}^{2}+2 \alpha_{2}^{2}+i h \alpha_{1}^{2}\left(s-\zeta s_{0}\right)\right]\right\} e^{i s h} \\
b_{2}=\frac{k_{1}^{2}}{s^{3}}\left\{(\zeta-1) \alpha_{2} s^{2}+\frac{i \kappa}{2}\left[s_{0}\left(s^{2}-\alpha_{2}^{2}\right)-i h s\left(\zeta s\left(s_{0}^{2}+\alpha_{2}^{2}\right)-s_{0}\left(s^{2}+\alpha_{2}^{2}\right)\right)\right]\right\} e^{i s h} .
\end{gathered}
$$

As a consequence of the above results, the coefficients $B_{1}$ and $B_{2}$ pertaining to the air region can be found as follows

$$
\begin{gathered}
B_{1}=-\zeta k_{1}^{2}\left\{\frac{2}{s+\zeta s_{0}}+i \kappa\left[\frac{2 \alpha_{2}}{\left(s+\zeta s_{0}\right)^{2}}+\frac{\alpha_{1}^{2} s_{0}}{\alpha_{2} s\left(s+s_{0}\right)\left(s+\zeta s_{0}\right)}+\frac{i h \alpha_{1}^{2}}{\alpha_{2}\left(s+\zeta s_{0}\right)}\right]\right\} e^{i s h} \\
B_{2}=-\alpha_{1} k_{1}^{2}\left\{\frac{2(\zeta-1)}{\left(s+s_{0}\right)\left(s+\zeta s_{0}\right)}+i \zeta \kappa\left[\frac{2 \alpha_{2}}{s\left(s+\zeta s_{0}\right)^{2}}-\frac{s_{0}^{2}+\alpha_{2}^{2}}{\alpha_{2} s\left(s+s_{\theta}\right)\left(s+\zeta s_{0}\right)}\right.\right. \\
\left.\left.-i h\left(\frac{k_{0}^{2}}{\alpha_{2} s\left(s+s_{0}\right)}-\frac{\alpha_{1}^{2}}{\alpha_{2} s\left(s+\zeta s_{0}\right)}\right)\right]\right\} e^{i s h} .
\end{gathered}
$$

In the above expressions the first terms represent the regular field in the absence of the magnetostatic field. The terms preceded by $\kappa$ represent the first order correction for the presence of the magnetostatic field.

To facilitate the approximate evaluation of the various integrals we shall define certain fundamental integrals from which all others could be derived by differentiation. We define

$$
\begin{gathered}
U_{1}=\frac{1}{k_{0} \pi} \int_{-\infty}^{\infty} \frac{e^{i s h}}{s+\zeta s_{0}} e^{i\left(\alpha_{1} x+\alpha_{2} y+s_{0} z\right)} d \alpha_{1} d \alpha_{2} \\
U_{2}=\frac{k_{0}}{2 \pi i} \int_{-\infty}^{\infty} \int_{-\infty} \frac{e^{i s h}}{s \alpha_{2}\left(s+\zeta s_{0}\right)} e^{i\left(\alpha_{1} x+\alpha_{2} y+s_{0} z\right)} d \alpha_{1} d \alpha_{2}
\end{gathered}
$$




$$
U_{3}=\frac{k_{0}}{\pi} \int_{-\infty}^{\infty} \int_{-\infty} \frac{e^{i s h}}{s\left(s+\zeta s_{0}\right)^{2}} e^{i\left(\alpha_{1} x+\alpha_{2} y+s_{0} z\right)} d \alpha_{1} d \alpha_{2} .
$$

The above integrals are not independent. Indeed, one can show that they are related as follows: ${ }^{5}$

$$
\begin{aligned}
U_{1} & =-\frac{2 i}{k_{0}^{2}} \partial_{h} \partial_{y} U_{2} \\
2 i \partial_{y} U_{2} & =\left(\partial_{h}+\zeta \partial_{z}\right) U_{3} .
\end{aligned}
$$

In what follows we shall avail ourselves of the above relationships to check the differentiability of the results after integration is performed. Employing the above definitions of $U_{1}, U_{2}$, and $U_{3}$ one can write the components of the Hertzian vector in terms of them. One obtains

$$
\begin{gathered}
\Pi_{x 0}^{(M)}=-\frac{\zeta m k_{0}}{4 \pi \omega \mu_{0}}\left\{U_{1}-\frac{i \kappa}{k_{0}^{2}}\left[\partial_{h} \partial_{y} U_{3}+i \partial_{x}^{2}\left(\frac{1}{(1-\zeta) k_{0}^{2}} \partial_{z}\left(\partial_{h}-\partial_{z}\right)+h \partial_{h}\right) U_{2}\right]\right\} \\
\Pi_{z 0}^{(M)}=-\frac{\zeta m \partial_{x}}{4 \pi k_{0} \omega \mu_{0}}\left(\left(\frac{1}{\zeta}\left(\partial_{h}-\partial_{z}\right) U_{1}+i \kappa\left\{\partial_{y} U_{3}-\frac{i}{(1-\zeta) k_{0}^{2}}\left(\partial_{y}^{2}+\partial_{z}^{2}\right)\left(\partial_{h}-\partial_{z}\right) U_{2}\right.\right.\right. \\
\left.\left.\left.+i h\left[\frac{1}{1-\zeta}\left(\partial_{h}-\partial_{z}\right)\left(\partial_{h}+\zeta \partial_{z}\right)+\partial_{x}^{2}\right] U_{2}\right\}\right)\right) .
\end{gathered}
$$

The integrals in (38) are surface integrals over the entire $\alpha_{1}-\alpha_{2}$ plane of a form that lends itself readily to a transformation to cylindrical coordinates in the configuration space as well as in the transform space. Thus we employ the transformation

for the space coordinates and

$$
\begin{aligned}
x & =\rho \cos \varphi \\
y & =\rho \sin \varphi \\
\alpha_{1} & =\lambda \cos \sigma \\
\alpha_{2} & =\lambda \sin \sigma
\end{aligned}
$$

for the transform variables. Now the fundamental integrals are of two distinct types. The first one is

$$
I_{1}=2 \pi \int_{0}^{\infty} F_{1}\left(\lambda^{2}\right) J_{0}(\lambda \rho) \lambda d \lambda
$$

The second distinct integral is of the form

$$
I_{2}=\int_{0}^{\infty} F_{2}\left(\lambda^{2}\right) d \lambda \int_{-\pi}^{\pi} \frac{e^{i \lambda \rho \cos (\sigma-\varphi)}}{\sin \sigma} d \sigma .
$$

In the appendix we show that

$$
\int_{-\pi}^{\pi} \frac{e^{i \lambda \rho \cos (\sigma-\varphi)}}{\sin \sigma} d \sigma=4 \pi i \sum_{0}^{\infty}(-1)^{\nu} \sin (2 \nu+1) \varphi J_{2 \nu+1}(\lambda \rho)
$$

which in connection with (44) gives

$$
I_{2}=4 \pi i \sum_{0}^{\infty}(-1)^{\nu} \sin (2 \nu+1) \varphi \int_{0}^{\infty} F_{2}\left(\lambda^{2}\right) J_{2 \nu+1}(\lambda \rho) d \lambda .
$$

In view of the above we can rewrite the fundamental integrals as follows:

$$
U_{1}=\frac{1}{k_{0}} \int_{-\infty}^{\infty} \frac{e^{i s h}}{s+\zeta s_{0}} e^{i s_{0} z} H_{0}^{(1)}(\lambda \rho) \lambda d \lambda
$$

${ }^{5}$ It may be shown that the differentiation under the integral sign is permissible by virtue of the fact that the resulting asymptotic expan sions are identical. 


$$
\begin{aligned}
& U_{2}=k_{0} \sum_{0}^{\infty}(-1)^{\nu} \sin (2 \nu+1) \varphi \int_{-\infty}^{\infty} \frac{e^{i s h}}{s\left(s+\zeta s_{0}\right)} e^{i s_{0} z} H_{2 \nu+1}^{(1)}(\lambda \rho) d \lambda \\
& U_{3}=k_{0} \int_{-\infty}^{\infty} \frac{e^{i s h}}{s\left(s+\zeta s_{0}\right)^{2}} e^{i s_{0} z} H_{0}^{(1)}(\lambda \rho) \lambda d \lambda
\end{aligned}
$$

where we used a well-known relationship between the Bessel and Hankel functions.

Now we transform to spherical coordinates using the transformation

$$
\begin{aligned}
& z=r \cos \theta \\
& \rho=r \sin \theta
\end{aligned}
$$

for the configuration space and

$$
\lambda=k_{0} \sin \beta
$$

for the transformation space. The results are

$$
\begin{aligned}
& U_{1}=\int_{\Gamma_{1}} \frac{\sin \beta \cos \beta \check{H}_{0}^{(1)}\left(k_{0} \rho \sin \beta\right)}{\sqrt{\zeta-\sin ^{2} \beta}+\zeta \cos \beta} e^{i k_{0} h \sqrt{\zeta-\sin 2} \beta} e^{i k_{0} r \cos (\beta-\theta)} d \beta \\
& U_{2}=\sum_{0}^{\infty}(-1)^{\nu} \sin (2 \nu+1) \varphi \int_{\Gamma_{1}} \frac{\cos \beta \check{H}_{2 \nu+1}^{(1)}\left(k_{0} \rho \sin \beta\right) e^{i k_{0} h \sqrt{\zeta-\sin ^{2} \beta}}}{\sqrt{\zeta \sin ^{2} \beta}\left(\sqrt{\zeta-\sin ^{2} \beta}+\zeta \cos \beta\right)} e^{i k_{0} r \cos (\beta-\theta)} d \beta \\
& U_{3}=\int_{\Gamma_{1}} \frac{\sin \beta \cos \beta \check{H}_{0}^{(1)}\left(k_{0} \rho \sin \beta\right) e^{i k_{0} h \sqrt{\zeta-\sin ^{2} \beta}}}{\sqrt{\zeta-\sin ^{2} \beta}\left(\sqrt{\zeta-\sin ^{2} \beta}+\zeta \cos \beta\right)^{2}} e^{i k_{0} r \cos (\beta-\theta)} d \beta
\end{aligned}
$$

where $\Gamma_{1}$ is the appropriate path of integration in the complex $\beta$-plane and

$$
\check{H}_{m}^{(1)}\left(k_{0} \rho \sin \beta\right)=H_{m}^{(1)}\left(k_{0} \rho \sin \beta\right) e^{-i k_{0} \rho \sin \beta} .
$$

In what follows we shall evaluate the integrals in (50) by the method of steepest descent which is contingent upon being able to deform the original path of integration $\Gamma_{1}$ to the path of steepest descent $\Gamma$. In the process of the continuous path deformation we must make sure that any singularities between these two paths are properly accounted for. To this end we shall investigate the location of the various singularities of the integrands of $U_{1}, U_{2}$, and $U_{3}$ in the complex $\beta$-plane.

The examination of the integrands in (50) reveals a possibility of poles at points where the denominator vanishes, i.e., where $\sin \beta= \pm(\zeta)^{\frac{1}{2}}(1+\zeta)^{-\frac{1}{2}}$. The corresponding Riemann surface consists of two sheets and we shall denote the upper sheet one on which $\operatorname{Im}\left\{\left(\zeta-\sin ^{2} \beta\right)^{\frac{1}{2}}\right\}>0$ and the lower sheet one on which $\operatorname{Im}\left\{\left(\zeta-\sin ^{2} \beta\right)^{\frac{1}{2}}\right\}<0$. Then it follows [Tyras, 1962] that when $|\sqrt{\zeta}|<1$ the resulting poles $P_{1}, P_{2}, P_{3}$, and $P_{4}$ are located in the complex $\beta$-plane as shown in figure 2 where $P_{1}$ and $P_{2}$ are the upper sheet poles and $P_{3}$ and $P_{4}$ are the lower sheet poles. In particular it can be shown [Tyras, 1962] that the poles $P_{3}$ and $P_{4}$ are on the same side of the branch cut $\operatorname{Im}\left\{\left(\zeta-\sin ^{2} \beta\right)^{\frac{1}{2}}\right\}=0$ as the origin.

The integrands in (50) also contain the radical $\left(\zeta-\sin ^{2} \beta\right)^{\frac{1}{2}}$ as a result of which the point $\beta= \pm \operatorname{arc} \sin \sqrt{\zeta}$ is a branch point. The convergence of the integrals is assured if the path of integration at least begins and ends on the upper sheet. The two sheets are joined along the lines $\operatorname{Im}\left\{\left(\zeta-\sin ^{2} \beta\right)^{\frac{1}{2}}=0\right.$ starting at the branch points. These lines are shown dashed in figure 2 .

We now investigate the possibility of replacing the original path of integration $\Gamma_{1}$ with the path $\Gamma$ corresponding to the path of the steepest descents through the saddle point $\beta=\theta$. Since the poles $P_{3}$ and $P_{4}$ lie on the lower sheet they need not be taken into account when deforming the path of integration $\Gamma_{1}$ into $\Gamma$. The upper sheet poles $P_{1}$ and $P_{2}$ need not be taken into account either since they will never be crossed lying beyond the region of interest, $-\pi / 2<\theta<\pi / 2$. 
Figure 2. The complex $\beta$-plane.

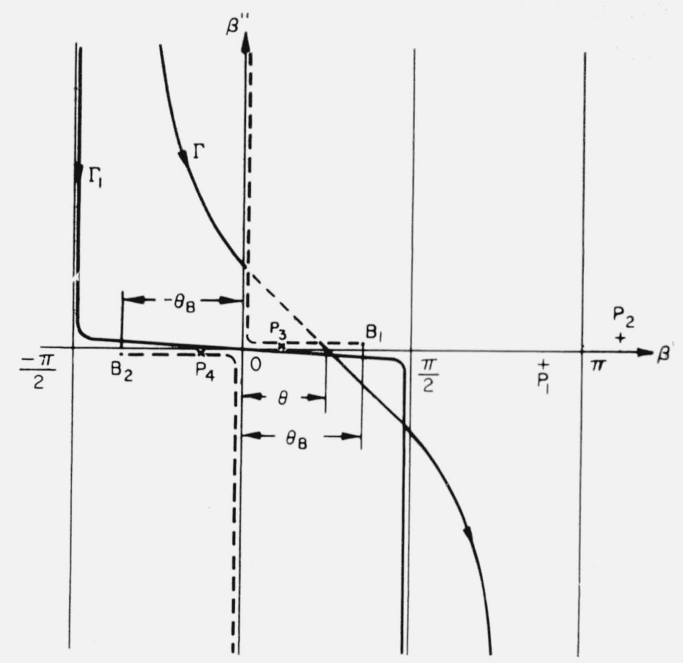

Next we focus our attention on the following. As we remarked earlier, the integrals in (50) are double valued since they contain the radical $\left(\zeta-\sin ^{2} \beta\right)^{\frac{1}{2}}$. The original path of integration passes over the upper sheet of the corresponding Riemann surface and it can be deformed into the path of the steepest descent $\Gamma$ only when at least the beginning and the end lie on this sheet. In the case when the angle $\theta$ does not exceed the critical angle $\theta_{B}=\operatorname{arc} \sin \sqrt{\zeta}$, the transition from the path $\Gamma_{1}$ to the path $\Gamma$ is accomplished without complications and the only contribution to the integrals will be from the saddle point. When $\theta$ exceeds the critical angle $\theta_{B}$ a more complicated path must be devised which results in the contribution from the saddle point and the contribution from the integration along the borders of the branch cut. The latter contribution results in the phenomenon of lateral waves [Brekhovskikh, 1960; Tyras et al., 1963]. In the present formulation we introduced the high frequency approximation in (33) excluding the neighborhood of the point $\zeta=\sin ^{2} \beta$ from the region of validity. This makes the integral expressions of (50) not suitable for carrying out the branch cut integration and thus the evaluation of the lateral wave field. ${ }^{6}$

The evaluation of the integrals in $(50)$ by the method of saddle-point integration is a straightforward process [Brekhovskikh, 1960]. We obtain for the leading term

$$
\begin{aligned}
& U_{1} \sim-\frac{i 2 \cos \theta e^{i k_{0} h \sqrt{\zeta-\sin ^{2} \theta}}}{\sqrt{\zeta-\sin ^{2} \theta}+\zeta \cos \theta} \cdot \frac{e^{i k_{0} r}}{k_{0} r} \\
& U_{3} \sim-\frac{i 2 \cos \theta e^{i k_{0} h \sqrt{\zeta-\sin ^{2} \theta}}}{\sqrt{\zeta-\sin ^{2} \theta}\left(\sqrt{\zeta-\sin ^{2} \theta}+\zeta \cos \theta\right)^{2}} \cdot \frac{e^{i k_{0} r}}{k_{0} r}
\end{aligned}
$$

The evaluation of the integral $U_{2}$ in $(50 \mathrm{~b})$ is more complicated and we shall use the saddlepoint method for double integration to accomplish it [Baños and Wesley, 1953]. First we put

where

$$
I=\int_{\Gamma_{\mathbf{1}}} F(\beta) e^{i k_{0} r \cos (\beta-\theta)} d \beta
$$

Now we put

$$
F(\beta)=\frac{\stackrel{\cos \beta \mathscr{H}_{2 \nu+1}^{(1)}}{(1)}\left(k_{0} \rho \sin \beta\right) e^{i k_{o} h \sqrt{\zeta-\sin ^{2} \beta}}}{\sqrt{\zeta-\sin ^{2} \beta}\left(\sqrt{\zeta-\sin ^{2} \beta}+\zeta \cos \beta\right)} .
$$

$$
w=\beta-\theta
$$

${ }^{6}$ It may be of interest to point out that in the rigorous formulation the branch points occur at $s_{1,2}=0$ corresponding to sin $\beta= \pm \sqrt{\epsilon \pm \eta}$. As $\omega_{H} / \omega \rightarrow 0$ these two branch points coalesce into one at $\sin \beta= \pm \sqrt{\zeta}$. The evaluation of the lateral field is possible in the case of line sources [Tyras et al., 1963]. 
which transfers the saddle-point to the point $w=0$ in the complex $w$-plane. Following the procedure of Baños and Wesley [1953] we make the next transformation as follows:

and note

$$
\frac{x^{2}}{2}=i k_{0} r(1-\cos w)
$$

$$
\frac{d w}{d x}=\frac{x}{i k_{0} r \sin w} .
$$

It can be shown that $w$ can be expanded in a power series of $x$ as follows:

$$
w=\frac{x}{\left(i k_{0} r\right)^{1 / 2}}+\frac{1}{24} \cdot \frac{x^{3}}{\left(i k_{0} r\right)^{3 / 2}}+\frac{3}{640} \cdot \frac{x^{5}}{\left(i k_{0} r\right)^{5 / 2}}+\ldots
$$

from which we note that $w$ is an odd function of $x$, i.e., $w(x)=-w(-x)$. Using the above transformation we can recast the integral (54) in the form

$$
I=e^{i k_{0} r} \int_{0}^{\infty} G(x) e^{-x^{2} / 2} d x
$$

where

$$
G(x)=\left[F(w+\theta) \stackrel{\vee}{H_{\nu}^{(1)}}\left(k_{0} \rho \sin (w+\theta)\right)+F(-w+\theta) \stackrel{\vee}{H_{\nu}^{(1)}}\left(k_{0} \rho \sin (-w+\theta)\right)\right] \frac{d w}{d x} .
$$

Putting this expression under the integral sign in (60) results in two integrals with the integration range from 0 to $+\infty$. In the second integral we make the substitution $x=-x^{\prime}$ and reverse the limits of integration. Due to the fact that $d w / d x$ is an even function of $x$, we can add these two integrals to form a single one as follows:

$$
I=\frac{e^{i k_{0} r}}{i k_{0} r} \int_{-\infty}^{\infty} \frac{x F(w+\theta)}{\sin w} \stackrel{\vee}{H_{\nu}^{(1)}}\left(k_{0} \rho \sin (w+\theta)\right) e^{-x^{2} / 2} d x .
$$

Now we make use of the integral representation of the Hankel function

$$
H_{\nu}^{(1)}(z)=\frac{4 e^{-i v} e^{i z}}{\sqrt{\pi} 2^{3 \nu} \Gamma(\nu+1 / 2) z^{\nu}} \int_{0}^{\infty} y^{2 \nu}\left(4 i z-y^{2}\right)^{\nu-1 / 2} e^{-y^{2} / 2} d y
$$

which we put in (62) to obtain

$$
I=\frac{4 e^{i\left(k_{0} r-\nu \pi\right)}}{i k_{0} r \sqrt{\pi} 2^{3 \nu} \Gamma(\nu+1 / 2)\left(k_{0} \rho\right)^{\nu}} \int_{-\infty}^{\infty} \int_{0}^{\infty} Q(x, y) e^{-x^{2 / 2}} e^{-y^{2} / 2} d x d y
$$

where

$$
Q(x, y)=\frac{x y^{2 \nu} F(w+\theta)\left[4 i k_{0} \rho \sin (w+\theta)-y^{2}\right]^{\nu-1 / 2}}{\sin w[\sin (w+\theta)]^{\nu}} .
$$

We recognize the integral (64) as being analogous in form to single integral of the type to which Watson's Lemma can be applied. A theoretical basis extending Watson's Lemma to double integrals has been provided by Baños and Wesley [1953]. Thus, at this point all that remains to be done to find the saddle-point contribution is to expand the integrand $Q(x, y)$ in a double power series and integrate term by term. When performing such an integration we avail ourselves of the following well-known result [Brekhovskikh, 1960]

$$
\int_{-\infty}^{\infty} x^{2 v} e^{-a x^{2} / 2} d x=\Gamma\left(\nu+\frac{1}{2}\right)\left(\frac{2}{a}\right)^{\nu+1 / 2}
$$

Combining the above results we obtain for the leading term

$$
U_{2} \sim \frac{-2 \cos \theta e^{i k_{0} h \sqrt{\zeta-\sin ^{2} \theta}} e^{i k_{0} r}}{\sin \theta \sqrt{\zeta-\sin ^{2} \theta}\left(\sqrt{\zeta-\sin ^{2} \theta}+\zeta \cos \theta\right) k_{0} r} \cdot \sum_{0}^{\infty} \sin (2 \nu+1) \varphi .
$$


The series on the right in (67) can be summed. We write formally

$$
\sum_{0}^{\infty} \sin (2 \nu+1) \varphi=\operatorname{Im}\left\{e^{i \varphi} \sum_{0}^{\infty} e^{i 2 \nu \varphi}\right\}=\frac{1}{2 \sin \varphi}
$$

where we used formula for summation of a binomial series. Thus

$$
U_{2} \sim \frac{-\cos \theta e^{i k_{0} h \sqrt{\zeta-\sin ^{2} \theta}}}{\sin \theta \sin \varphi \sqrt{\zeta-\sin ^{2} \theta}\left(\sqrt{\zeta-\sin ^{2} \theta}+\zeta \cos \theta\right)} \cdot \frac{e^{i k_{0} r}}{k_{0} r} .
$$

Collecting the above results we find the components of the Hertzian vector and hence the field components. Thus

$$
\begin{aligned}
& E_{\theta_{0}} \sim-\frac{i k_{0} m \cos \theta e^{i k_{0} h \sqrt{\zeta-\sin ^{2} \theta}}}{2 \pi\left(\zeta \cos \theta+\sqrt{\zeta-\sin ^{2} \theta}\right)}\left\{\zeta \sin \varphi+i \eta \sin \theta\left[\frac{\sin ^{2} \varphi}{\zeta \cos \theta+\sqrt{\zeta-\sin ^{2} \theta}}\right.\right. \\
& \left.\left.+\frac{\cos ^{2} \varphi \cos \theta}{2 \sqrt{\zeta-\sin ^{2} \theta}\left(\cos \theta+\sqrt{\zeta-\sin ^{2} \theta}\right)}+\frac{i k_{0} h \cos ^{2} \varphi}{2}\right]\right\} \frac{e^{i k_{0} r}}{r} \\
& E_{\varphi 0} \sim \frac{-i k_{0} m \cos \varphi \cos \theta e^{i k_{0} h \sqrt{\zeta-\sin ^{2} \theta}}}{2 \pi\left(\zeta \cos \theta+\sqrt{\zeta-\sin ^{2} \theta}\right)}\left\{\sqrt{\zeta-\sin ^{2} \theta}\left(\cos \theta \sqrt{\zeta-\sin ^{2} \theta}+\sin ^{2} \theta\right)\right. \\
& +i \eta \frac{\sin \theta \sin \varphi}{\sqrt{\zeta-\sin ^{2} \theta}}\left[\frac{\sin ^{2} \theta+\cos \theta \sqrt{\zeta-\sin ^{2} \theta}}{\zeta \cos \theta+\sqrt{\zeta-\sin ^{2} \theta}}-\frac{1}{2\left(\cos \theta+\sqrt{\zeta-\sin ^{2} \theta}\right)}\right. \\
& \left.\left.-\frac{i k_{0} h\left(\zeta \cos \theta+\sqrt{\zeta-\sin ^{2} \theta}\right)}{2\left(\cos \theta+\sqrt{\zeta-\sin ^{2} \theta}\right)}\right]\right\} \frac{e^{i k_{0} r}}{r}
\end{aligned}
$$

$E_{r 0} \sim 0$.

One observes that the above field components contain the regular field that would exist in absence of the magnetostatic field and a first order correction for the presence of the magnetostatic field (the terms preceded by $\eta$ ). It will be seen that these corrections are not in general symmetric about the $\theta=0$ and $\varphi=0$ axes. The time average Poynting vector given by

$$
S_{r 0}=\frac{1}{2 Z_{0}}\left(\left|E_{\theta 0}\right|^{2}+\left|E_{\varphi 0}\right|^{2}\right) \mid
$$

where $Z_{0}$ is the free space impedance, is plotted in figures 3 and 4 when $\varphi=0$ and $\pi / 2$ respectively for a plasma roughly equivalent to that in the lower edge of the ionosphere and the magnetostatic field equivalent to that of the earth. It will be noted that the correction due to the magnetostatic field is noticeable only in the plane $\varphi=0$ and within the region of validity of (29) it is significant only in the proximity of the interface.

Figure 3. Power pattern in air of a horizontal magnetic dipole in magnetoplasma; $\varphi=0^{\circ}, \mathrm{N}=750$ electrons per cubic centimeter, $\mathrm{h}=1000$ meters, $\nu=10^{6}$ collisions per second.

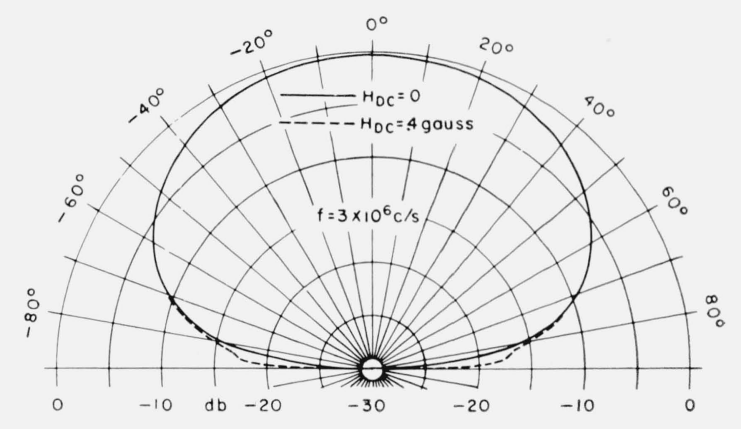




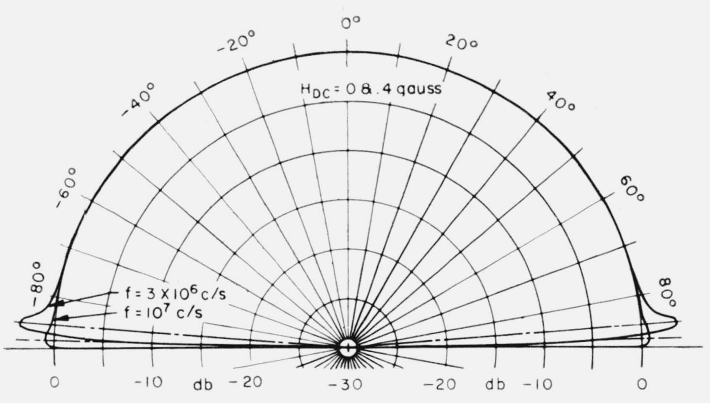

3. Dipole in Air

\subsection{Rigorous Formulation}

Since this problem is in many respects similar to the preceding one, we shall not go into it in great detail.

The geometry of the present problem is shown in figure 5. As before we shall work with the Hertzian vector of the magnetic type in the air region and with the actual field components in the magnetoplasma region.

For the air region we can write immediately

$$
\begin{aligned}
& \Pi_{x_{0}}^{(M)}=\frac{-m}{8 \pi^{2} \omega \mu_{0}} \int_{-\infty}^{\infty} \int_{-\infty}\left[\frac{e^{i s_{0}|z-h|}}{s_{0}}+B_{1} e^{i s_{0} z}\right] e^{i\left(\alpha_{1} x+\alpha_{2} y\right)} d \alpha_{1} d \alpha_{2} \\
& \Pi_{z_{0}}^{(M)}=\frac{-m}{8 \pi^{2} \omega \mu_{0}} \int_{-\infty}^{\infty} \int_{-\infty} B_{2} e^{i\left(\alpha_{1} x+\alpha_{2} y+s_{0} z\right)} d \alpha_{1} d \alpha_{2}
\end{aligned}
$$

whereas for the magnetoplasma region the integral representation of the field components is identical to that in (23). Application of the boundary conditions (25) gives a system of equations as follows:

where

$$
\left(\begin{array}{rlll}
-1 & 0 & C_{13} & C_{14} \\
0 & C_{22} & C_{23} & C_{24} \\
0 & C_{32} & C_{33} & C_{34} \\
1 & 0 & C_{34} & C_{44}
\end{array}\right) \quad\left(\begin{array}{c}
B_{1} \\
B_{2} \\
\chi A_{1} \\
\chi A_{2}
\end{array}\right)=\left(\begin{array}{l}
b \\
0 \\
0 \\
b
\end{array}\right)
$$

$$
\begin{aligned}
& C_{13}=\frac{\alpha_{2}\left[\Phi_{1}\left(s_{1}\right)+\alpha_{1}^{2} \Phi_{2}\left(s_{1}\right)\right]+i \kappa \chi \zeta k_{0}^{2} s_{1} \alpha_{1}^{2}}{\alpha_{2} \Phi_{1}\left(s_{1}\right)} \\
& C_{14}=C_{13}\left(s_{1}=s_{2}\right) \\
& C_{22}=\frac{s_{0}}{\alpha_{1}} \\
& C_{23}=\frac{\alpha_{2}\left[\Phi_{1}\left(s_{1}\right)-\left(k_{0}^{2}-\alpha_{1}^{2}\right) \Phi_{2}\left(s_{1}\right)\right]-i \kappa \chi \zeta k_{0}^{2}\left(k_{0}^{2}-\alpha_{1}^{2}\right) s_{1}}{\alpha_{2} \Phi_{1}\left(s_{1}\right)} \\
& C_{24}=C_{23}\left(s_{1}=s_{2}\right)
\end{aligned}
$$




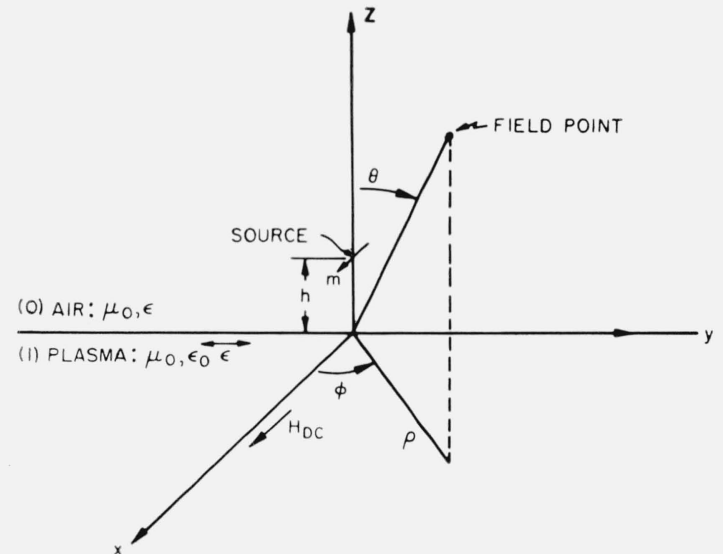

Figure 5. Geometry of the problem of magnetic dipole above a magnetoplasma halfspace.

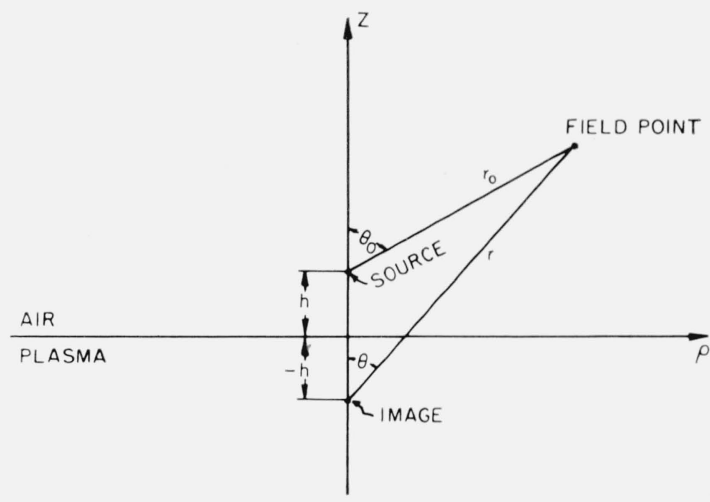

Figure 6. Geometry of the source and the image.

$$
\begin{aligned}
C_{32} & =\frac{\zeta}{\alpha_{1}} \\
C_{33} & =\frac{i \kappa \chi \zeta k_{0}^{2}\left(s_{1}^{2}+\alpha_{1}^{2}\right)}{\alpha_{2} \Phi_{1}\left(s_{1}\right)} \\
C_{34} & =C_{33}\left(s_{1}=s_{2}\right) \\
C_{43} & =\frac{\zeta \alpha_{2} \Phi_{1}\left(s_{1}\right)\left(s_{1}-i \kappa \alpha_{2}\right)+\alpha_{1}^{2}\left(\chi s_{1}-i \kappa \alpha_{2} \zeta\right)\left[\alpha_{2} \Phi_{2}\left(s_{1}\right)+i \kappa \chi \zeta k_{0}^{2} s_{1}\right]}{s_{0} \alpha_{2} \zeta \chi \Phi_{1}\left(s_{1}\right)}-\frac{\alpha_{1}^{2}(\chi-\zeta)\left[s_{1} \Phi_{1}\left(s_{1}\right)-i \kappa \zeta k_{0}^{2} \alpha_{2}\right]}{\zeta \chi \Phi_{1}\left(s_{1}\right)} \\
C_{44} & =C_{43}\left(s_{1}=s_{2}\right) \\
b & =\frac{e^{i s_{0} h}}{s_{0}} .
\end{aligned}
$$

The coefficients $A_{1,2}$ and $B_{1,2}$ are thus determinable from (76) but as before we shall forego finding their explicit form in the present formulation.

\subsection{High Frequency Approximation}

Using the approximations (33) we can find the coefficients $B_{1,2}$ as follows:

$$
\begin{aligned}
& B_{1}=\left\{-\frac{1}{s_{0}}+\frac{2 \zeta}{s+\zeta s_{0}}+i \eta\left[\frac{2 \alpha_{2}}{\left(s+\zeta s_{0}\right)^{2}}+\frac{\alpha_{1}^{2} s_{0}}{\alpha_{2} s\left(s+s_{0}\right)\left(s+\zeta s_{0}\right)}\right]\right\} e^{i s_{0} h} \\
& B_{2}=-\left\{\frac{2(\zeta-1) \alpha_{1}}{\left(s+s_{0}\right)\left(s+\zeta s_{0}\right)}+i \eta\left[\frac{2 \alpha_{1} \alpha_{2}}{s\left(s+\zeta s_{0}\right)^{2}}-\frac{\left.\alpha_{1}\left(s_{0}^{2}+\alpha_{2}^{2}\right)\right\}}{\alpha_{2} s\left(s+s_{0}\right)\left(s+\zeta s_{0}\right)}\right]\right\} e^{i s_{0} h} .
\end{aligned}
$$

To evaluate the field components we avail ourselves of the integrals $U_{1}, U_{2}$, and $U_{3}$ in (38) and the results in (52), (53), and (69) and obtain 


$$
\begin{aligned}
& E_{\theta 0} \sim \frac{-i m k_{0}}{2 \pi}\left\{\frac{\sin \varphi}{2}\left(\frac{e^{i k_{0} r}}{r}-\frac{e^{i k_{0} r_{0}}}{r_{0}}\right)+\frac{\zeta \cos \theta \sin \varphi}{\zeta \cos \theta+\sqrt{\zeta-\sin ^{2} \theta}} \cdot \frac{e^{i k_{0} r}}{r}\right. \\
& \left.\quad+\frac{i \eta \sin \theta \cos \theta}{\zeta \cos \theta+\sqrt{\zeta-\sin ^{2} \theta}}\left[\frac{\sin ^{2} \varphi}{\zeta \cos \theta+\sqrt{\zeta-\sin ^{2} \theta}}+\frac{\cos \theta \cos ^{2} \varphi}{2 \sqrt{\zeta-\sin ^{2} \theta}\left(\cos \theta+\sqrt{\zeta-\sin ^{2} \theta}\right)}\right] \frac{e^{i k_{0} r}}{r}\right\} \\
& E_{\varphi 0} \sim \frac{-i k_{0} m \cos \varphi \cos \theta}{2 \pi}\left\{\frac{1}{2}\left(\frac{e^{i k_{0} r}}{r}-\frac{e^{i k_{0} r_{0}}}{r_{0}}\right)+\frac{\sqrt{\zeta-\sin ^{2} \theta}\left(\sin ^{2} \theta+\cos \sqrt{\zeta-\sin ^{2} \theta}\right)}{\zeta \cos \theta+\sqrt{\zeta-\sin ^{2} \theta}} \cdot \frac{e^{i k_{0} r}}{r}\right. \\
& \left.+\frac{i \eta \sin \theta \sin \varphi}{\sqrt{\zeta-\sin ^{2} \theta}\left(\zeta \cos \theta+\sqrt{\zeta-\sin ^{2} \theta}\right)}\left[\frac{\sin ^{2} \theta+\cos \theta \sqrt{\zeta-\sin ^{2} \theta}}{\zeta \cos \theta+\sqrt{\zeta-\sin ^{2} \theta}}-\frac{1}{2\left(\cos \theta+\sqrt{\zeta-\sin ^{2} \theta}\right)}\right] \frac{e^{i k_{0} r}}{r}\right\} \\
& E_{r 0} \sim 0
\end{aligned}
$$

where $r_{0}$ and $r$ are the distances from the source and its image to the field point respectively as shown in figure 6 .

\section{Appendix}

The integral under consideration is

$$
I=\int_{-\pi}^{\pi} \frac{e^{i \lambda \rho \cos (\sigma-\varphi)}}{\sin \sigma} d \sigma .
$$

Expanding the numerator in terms of Bessel functions [Stratton, 1941] and interchanging the order of summation and integration gives

$$
I=\sum_{-\infty}^{\infty} i^{n} e^{-i n \varphi} J_{n}(\lambda \rho) \int_{-\pi}^{\pi} \frac{e^{i n \sigma}}{\sin \sigma} d \sigma .
$$

Now we focus our attention on the integral on the right. By making the transformation $z=e^{i \sigma}$ one obtains

$$
I_{1}=\int_{-\pi}^{\pi} \frac{e^{i n \sigma}}{\sin \sigma} d \sigma=2 \int_{c} \frac{z^{n} d z}{(z+1)(z-1)}
$$

where $C$ is a unit circle. Now we consider

$$
K=\int_{\Gamma} \frac{z^{n} d z}{(z+1)(z-1)}
$$

where the contour is shown in figure 7 . Integrating formally it can be shown that

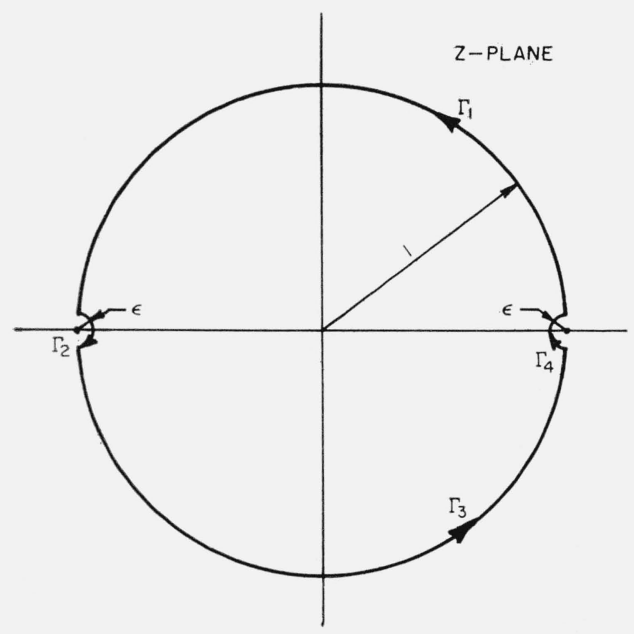

Figure 7. The complex z-plane. 
which gives

$$
I_{1}=-2 \lim _{\epsilon \rightarrow 0}\left\{\int_{\Gamma_{2}}+\int_{\Gamma_{4}}\right\}
$$

$$
I_{1}=\pi i\left[1-(-1)^{n}\right] \text {. }
$$

Substituting the (A6) into (A2) we obtain the desired result

$$
\int_{-\pi}^{\pi} \frac{e^{i \lambda \rho \cos (\sigma-\varphi)}}{\sin \sigma} d \sigma=4 \pi i \sum_{0}^{\infty}(-1)^{n} \sin (2 n+1) \varphi J_{2 n+1}(\lambda \rho) .
$$

\section{References}

Arbel, E. (Nov. 1960), Radiation from a point source in an anisotropic medium, Research Report PIEMRI 861-60, Microwave Research Institute, Polytech. Inst., Brooklyn, N.Y.

Baños, A., and J. P. Wesley (Sept. 1953), The horizontal electric dipole in a conducting halfspace, Marine Physical Laboratory of the Scripps Institution of Oceanography, Univ. of Calif., La Jolla, Calif.

Barsukov, K. A. (1959), Radiation of electromagnetic waves from a point source in a gyrotropic medium with separation boundary, Radio Engrs. Electron. 4, 1-9.

Brekhovskikh, L. M. (1960), Waves in layered media (Academic Press, New York and London).

Ince, E. L. (1956), Ordinary differential equation (Dover Publication, New York, N.Y.).

Moore, R. K. (1951), The theory of radio communication between submerged submarines, Doctoral Dissertation (Cornell University, Ithaca, N.Y.).

Ratcliffe, J. A. (1959), The magnetoionic theory and its applications to the ionosphere (University Press, Cambridge, London).

Sommerfeld, A. (1926), Über die Ausbreitung der Wellen in der drahtlosen Telegraphie, Ann. Physik 81, 1135-1153.

Stratton, J. A. (1941), Electromagnetic theory (McGraw-Hill Book Co., Inc., New York, N.Y.).

Tyras, G., and G. Held (Dec. 1959), On the propagation of electromagnetic waves through anisotropic layers, IRE Trans. Ant. Prop. AP-y, Special Supplement, S296-S300.

Tyras, G. (1962), Radiation from sources in magnetoplasma with a separation boundary, Doctoral Dissertation (University of Washington, Seattle, Wash.).

Tyras, G., A. Ishimaru, and H. M. Swarm (1963, to be published), Lateral waves on air magnetoplasma interfaces, Symposium on Electromagnetic Theory and Antennas, 1962 Copenhagen.

Wait, J. R., and L. L. Campbell (June 1953), The fields of an oscillating magnetic dipole immersed in a semiinfinite conducting medium, J. Geophys. Res. 58, 167-178.

Wait, J. R. (1959), Radiation from a small loop immersed in a semi-infinite conducting medium, Can. J. Phys. 37, $672-674$.

(Paper 67D5-282) 